\title{
WNT4, a potential biomarker in serum for colorectal cancer, promotes metastasis through WNT4/ $\beta$-catenin pathway
}

\section{Dongmei Yang}

Renmin hospital of Wuhan University

\section{Qing Li}

Renmin hospital of Wuhan University

\section{Renduo Shang}

Renmin hospital of Wuhan University

\section{Liwen Yao}

Renmin hospital of Wuhan University

\section{Lianlian Wu}

Renmin hospital of Wuhan University

\section{Mengjiao Zhang}

Renmin Hospital of Wuhan University

\section{Lihui Zhang}

Renmin hospital of Wuhan University

\section{Ming Xu}

Renmin hospital of Wuhan University

\section{Zihua Lu}

Renmin hospital of Wuhan University

Jie Zhou

Renmin hospital of Wuhan University

Li Huang

Renmin hospital of Wuhan University

\section{Xiaodong Huang}

Renmin hospital of Wuhan University

\section{Du Cheng}

Renmin hospital of Wuhan University

\section{Yanning Yang}

Renmin hospital of Wuhan University

Honggang Yu ( $\nabla$ yuhonggang@whu.edu.cn )

Renmin hospital of Wuhan University

\section{Research}

Keywords: WNT4, biomarker, colorectal cancer, tumor metastasis, epithelial-mesenchymal transition, fibroblast, angiogenesis

Posted Date: August 20th, 2020

DOl: https://doi.org/10.21203/rs.3.rs-62264/v1

License: (c) (1) This work is licensed under a Creative Commons Attribution 4.0 International License. Read Full License 
Version of Record: A version of this preprint was published on November 23rd, 2020. See the published version at https://doi.org/10.1186/s13046-020-01774-w. 


\section{Abstract \\ Background}

Wingless and Int-related protein (Wnt) ligands were aberrantly expressed in human diseases. However, the aberrant level of Wnt ligands have not been explored in serum. Here, we aimed to identify the WNT4 level in serum and explore its oncogenic role in colorectal cancer (CRC).

\section{Methods}

Serum samples from $40 \mathrm{CRC}$ patients and 28 healthy donors were collected to measure the levels of WNT4. CRC tissue samples from patients were collected to explore the resource of WNT4. Further, we used CRC cells and xenograft mouse model to explore the oncogenic role of WNT4.

\section{Results}

WNT4 was significantly upregulated in serum of colorectal cancer (CRC) patients, and CRC tissues were identified as an important source of elevated WNT4 levels in CRC patients. Interestingly, the elevated WNT4 in serum was downregulated after tumor resection. Next, we found that WNT4 could contributed to epithelial-to-mesenchymal transition and activated fibroblasts by activating WNT4/ $\beta$-catenin pathway in vitro and in vivo; besides, angiogenesis was also induced via WNT4/ $\beta$ catenin/Ang2 pathway. Those effects could be reversed by ICG-001, a $\beta$-catenin/TCF inhibitor.

\section{Conclusion}

These data indicated that WNT4 may be a potential biomarker for CRC and provided evidence to support a critical role of WNT4 in promoting CRC cell metastasis by inducing epithelial-to-mesenchymal transition, activating fibroblasts and promoting angiogenesis in the colorectal tumor microenvironment.

\section{Introduction}

Colorectal cancer (CRC) is one of the leading causes of cancer-related death worldwide [1], which evolves as a result of the accumulation of genetic events and epigenetic events [2]. The data evaluated by the International Agency for Research on Cancer (IARC) indicated that about 1.8 million new cases of CRC was diagnosed and over 860,000 CRC patients were died worldwide in 2018 , which account for about $10 \%$ of all cancers and $9 \%$ of all cancer-related deaths, respectively [3]. Therefore, it is urgently needed to develop sensitive biomarkers and effective treatments for patients with CRC.

Nowadays, More and more attention has been paid to the research of biomarkers for diseases, which could be used to early diagnose and predict outcome of cancers [4]. Besides, measuring biomarkers in blood samples offers a simple and effective way for illness diagnosis [5]. Among nineteen identified WNT ligands, WNT4 is highly secretory and its secretion has been reported in wound healing, acute kidney injury, and angiogenesis [6]. However, to our knowledge, there is no study on exploring Wnt levels in blood samples. Therefore, it would be interesting and meaningful to explore the levels of Wnt proteins in blood samples.

Wnt/ $\beta$-catenin pathway is the "canonical" Wnt pathway, which is essential for stem cell self-renewal and maintaining the homeostasis of intestinal tract [7]. As a member of Wnt family, WNT4 may also play a role in CRC through $\beta$-catenindependent pathway.

The tumor stroma is composed of extra-cellular matrix (ECM) proteins and a variety of cell types including fibroblasts, infiltrating immune cells, and endothelial cells $[8,9]$. Fibroblasts are a major component of tumor stroma and these cells may 
acquire an activated phenotype called cancer associated fibroblasts (CAF), which specifically expressed markers like fibronectin (FN) and a-SMA $[10,11]$. CAF has been demonstrated to have the ability to modulate the tumor microenvironment to promote CRC [12]. Cytokines and inflammatory factors derived from cancer tissue can stimulate the transformation of normal fibroblast (NF) into CAF [13]. Therefore, searching for the activators of CAF in CRC may play a potential role in inhibiting the development of CRC.

In addition, tumor start to grow only when the endothelial cells are recruited to the tumor site and form capillaries [14]. Previous studies have demonstrated that angiogenesis could be enhanced by WNT4/ $\beta$-catenin pathway in Human umbilical cord mesenchymal stem cell [15]. ANG2 is one of the crucial genes that is vital for angiogenesis and closely related to functions of human umbilical vein endothelial cells (HUVECs) [16]. However, it is not clear that whether WNT4/ $\beta$-catenin pathway could affect angiogenesis in CRC by regulating ANG2.

In this study, we first identified that WNT4 was increased in serum samples of CRC patients and originated from colorectal cancer tissues, which was decreased after tumor resection. Mechanistically, we explored its role in the development of CRC in vitro and in vivo, we found that WNT4 could promote epithelial-to-mesenchymal transition (EMT) of CRC cells, triggers NFs transformed into CAFs, and induce angiogenesis in tumor in a $\beta$-catenin-dependent pathway.

\section{Materials And Methods}

\section{Human colorectal tissue and serum samples}

The CRC tissues and paired adjacent normal tissues were collected from 16 CRC patients undergoing surgical resection, serum samples from 40 CRC patients and 28 healthy donors were also collected at the Renmin Hospital of Wuhan University, Wuhan, China. Informed consents were signed by those patients and healthy donors before sample collection. All works were approved by the ethics review board of Renmin Hospital of Wuhan University and carried out in compliance with the Declaration of Helsinki of the World Medical Association.

\section{Oncomine Database Analysis}

The Oncomine gene expression array database (www.oncomine.org) was used to assess mRNA levels of WNT4 in two CRC datasets, including Sabates-Bellver Colon (http://www.ncbi.nlm.nih.gov/geo/query/acc.cgi?acc=GSE8671) and Skrzypczak Colorectal (http://www.ncbi.nlm.nih.gov/geo/query/acc.cgi?acc=GSE20916). The Cancer Genome Atlas (TCGA; http://tcgadata.nci.nih.gov/tcga/) Colorectal and Jorissen Colorectal 3 (http://www.ncbi.nlm.nih.gov/geo/query/acc.cgi? acc=GSE14333) were applied to analyze survival rates and recurrence rates respectively. Samples with incomplete clinical information were excluded.

\section{Antibodies and reagents}

Antibodies were obtained from the following commercial sources: rabbit anti-WNT4 polyclonal antibody (ab91226), rabbit anti-AXIN2 monoclonal antibody (ab109307), rabbit anti-fibronectin polyclonal antibody (ab2413) and rabbit anti- $\beta$-catenin monoclonal antibody (ab32572) were obtained from Abcam (MA, USA); rabbit anti-ZO-1 monoclonal antibody (\#13663) and rabbit anti-E-cadherin monoclonal antibody (\#3195) were obtained from Cell Signaling Technology (MA, USA); anti-rabbit and anti-mouse HRP-conjugated secondary antibodies (\#31460, \#31430) were obtained from Invitrogen (CA, USA). Antifluorescein isothiocyanate labeled phalloidin (P5282) and anti-a-SMA mouse monoclonal (A5228) antibodies were obtained from Sigma-Aldrich (St. Louis, MO, USA). Recombinant Human WNT4 Protein (6076-WN-005/CF) was obtained from R\&D Systems (Minneapolis, MN, USA).

\section{Cell culture}

The CRC cell line HCT 116, LoVo and SW480 were cultured in Dulbecco's modifed Eagle medium (DMEM, Gibco, Carlsbad, CA, USA) supplemented with $10 \%$ FBS, $100 \mathrm{U} / \mathrm{mL}$ penicillin, and $100 \mathrm{mg} / \mathrm{mL}$ streptomycin. HUVECs were cultured in Ham's F-12K 
(HyClone, Thermo Fisher Scientific, Waltham, MA, USA) supplemented with 10\% FBS, $0.1 \mathrm{mg} / \mathrm{mL}$ Heparin, 0.03-0.05 mg/ml endothelial cell growth supplement (ECGs). All cells were incubated in a humidified incubator containing $5 \% \mathrm{CO}_{2}$ at $37^{\circ} \mathrm{C}$.

\section{Immunohistochemical staining}

Indirect immunohistochemical staining was performed as follows. All sections were dewaxed and dehydrated, followed by antigen retrieval and blocking. Subsequently, sections were incubated with a primary antibody and HRP-conjugated secondary antibodies. A 3,3'-diaminobenzidine (DAB) kit was used for visualization, and hematoxylin was used to stain the nuclei. All the sections were dehydrated with alcohol and sealed with neutral resin.

\section{Cell migration and invasion assay}

Cell migration assay was performed with transwell chambers $(8.0 \mu \mathrm{m})$ in a 24-well plate. For migration assays, the cells were pretreated with $100 \mathrm{ng} / \mathrm{mL}$ WNT4 for 24 hours like the previous study, and then added to the upper chamber, which contained serum-free DMEM; the lower compartment was filled with DMEM medium supplemented with $10 \%$ FBS. Cells were allowed to migrate for $12 \mathrm{~h}$, and those that had migrated across the filter were fixed in methanol and stained with $0.1 \%$ crystal violet. Their invasion potential was determined by using a transwell chamber $(8.0 \mu \mathrm{m})$ coated with Matrigel (Corning, NY, USA) solution diluted in DMEM medium (1:4 v/v), and the lower compartment was filled with DMEM medium supplemented with $20 \%$ FBS. Cells were incubated for $24 \mathrm{~h}$ before the invasive cells were fixed, stained, and counted. Results were analyzed by ImageJ.

\section{ELISA}

WNT4 concentration in serum from healthy donors, CRC patients and conditioned medium were quantitatively determined using ELISA kits (CSB-EL026137HU, CUSABIO, Wuhan, China) according to the manufacturer's instructions. For ANG2, the protein concentrations in media from co-culture system were detected by a human ANG2 ELISA kit (Abcam, USA).

\section{Luciferase assay}

A Dual-Luciferase Reporter Assay system was used to measure the luciferase activities (Promega, WI, USA). For the TOP/FOPFlash reporter assays, cells were divided into five groups: blank, $100 \mathrm{ng} / \mathrm{mL}$ WNT4, DMSO, ICG-001, ICG-001+100 ng/mL WNT4. After transfection, WNT4 protein $(100 \mathrm{ng} / \mathrm{mL})$ was added $6 \mathrm{~h}$ before luciferase detection. The relative ratio of TOPFlash firefly luciferase activity to pRL-TK Renilla luciferase activity was recorded as the strength of the transcriptional activity.

\section{Western blots}

In brief, equal amounts of protein were run on SDS-PAGE, and transferred to PVDF (Millipore, MA, USA). After blocking with $5 \%$ non-fat milk in Tris-bufered saline with Tween 20 (TBST), the blots were incubated with the primary antibodies at $4^{\circ} \mathrm{C}$ overnight and HRP-conjugated secondary antibodies at room temperature for 60 minutes. Then the signals were detected by enhanced chemiluminescence $(E C L)$ reagents. The signals were detected by chemiluminescent gel imaging system.

\section{Immunofluorescence}

A 12-well chamber was used for cellular immunofluorescence assays. Each well was seeded with $1 \times 10^{5}$ cells on the coverslip and fixed with $4 \%$ paraformaldehyde冈treated with $0.2 \%$ Triton X-100 for 1 min and blocked with $1 \%$ bovine serum albumin (BSA). For paraffin embedded tissue slides, all sections were dewaxed and dehydrated, followed by antigen retrieval and blocking. After incubation with primary antibodies and fluorescent-labeled secondary antibodies and Phalloidin; the cells or tissue sections were stained with 4',6-diamidino-2-phenylindole (DAPI) and covered with a coverslip. The immunofluorescence was observed through an upright Olympus fluorescence microscope (OLYMPUS BX53).

\section{Transient transfection and establishment of stable expressing cells}


Inhibition of WNT4 expression was performed by using specific siRNA. The sequences of WNT4 siRNA-1, WNT4 siRNA-2 and normal control used in this study are presented in Supplementary table 1. The cells were transiently transfected with WNT4 siRNAs using Lipofectamine 2000 and were harvested for the analysis of the efficiency of knockdown 48 hours after transfection.

To establish stable WNT4-overexpressing cells, an overexpressing plasmid was constructed and packaged into a lentivirus system (WNT4-HA). Lentivirus-NC was used as the negative control (WNT4-vector). In addition, the short hairpin RNA (shRNA) sequence against WNT4 and ANG2 were transfected to generate cells with low expression of WNT4 (sh-WNT4) and ANG2 (shANG2) (Supplementary table 1), while the Scramble was used as control. Non-transfected cells were screened out by treatment with $10 \mu \mathrm{g} / \mathrm{mL}$ puromycin (Sigma Aldrich, St Louis, MO).

\section{Quantitative real-time PCR}

TRIzol reagents (Invitrogen) was used to extract RNA from cell lines or tissues according to the manufacturer's instructions. RT-qPCR was performed to quantify mRNA expression with SYBR Green PCR Master Mix (Takara, Ohtsu, Japan), and miRNA expression with the NCode miRNA RT-qPCR analysis (TaKaRa, Ohtsu, Japan). The related primer sequences are presented in

\section{Supplementary table 2.}

\section{Tumor xenograft study}

To generate a xenograft model, $1 \times 10^{7}$ cells $/ 200 \mu \mathrm{L}$ were injected subcutaneously into the dorsal flank of each nude mouse (8-week-old, male, BALB/c-nu/nu). After 2 weeks, the nude mice were sacrificed, and subcutaneous nodules were collected. For establishing the tumor metastasis model, $1 \times 10^{6}$ cells $/ 100 \mu \mathrm{L}$ were injected into the tail vein of each nude mouse (8week-old, male, BALB/c-nu/nu). After 4 weeks, the nude mice were sacrificed, and the weights of the mouse livers were measured.

All tissues were fixed with $4 \%$ paraformaldehyde after the tumor weights were measured. All animal experiments were approved by the Committee on the Use of Live Animals in Teaching and Research (CULATR), Renmin Hospital of Wuhan University (No:11400700326686 and No:110011111003022). All animals care was in accordance with institution guidelines.

\section{Isolation of NFs and CAFs from CRC and normal tissues}

Fresh tissues were cut into small pieces (around $1 \mathrm{~mm}^{3}$ ) and subjected to enzymatic digestion in DMEM supplemented with $5 \%$ fetal bovine serum following the instructions of the tumor dissociation kit (Miltenyi Biotec, Bergisch Gladbach, Germany). Cells were then filtered through a 100- $\mu \mathrm{m}$ cell strainer (Thermo Fisher Scientific, MA, USA) and resuspended and cultured in fibroblast medium (FM). Flow cytometry with CD31-Cy7 conjugated antibody, CD45-Cy7 conjugated antibody, and CD329-Cy7 conjugated antibody was performed to confirm the absence of endothelial, immune, and epithelial cell contamination in the primary fibroblasts.

\section{Preparation of the Conditioned Medium}

Fresh human CRC and adjacent normal tissue samples were obtained directly from the operating room. Tissues were weighed and preserved in Falcon tubes with $10 \mathrm{~mL}$ of DMEM with a high concentration of antibiotics $(100 \mathrm{U} / \mathrm{mL}$ penicillin, and 100 $\mathrm{mg} / \mathrm{mL}$ streptomycin) to avoid bacterial or fungal contamination, those tissue next were cut into similar sized pieces and cultured with DMEM individually for $12 \mathrm{~h}$. Then, the collected conditioned medium (CM) was filtered through 40- $\mu \mathrm{m}$ cell strainers (Thermo Fisher Scientific, MA, USA) to obtain tissue-conditioned medium and stored at $-80^{\circ} \mathrm{C}$ until further investigation.

After the SW480 cells (vector/WNT4-HA) grew to 70-80\% confluency, the cells were cultured in DMEM medium (no FBS) for 24h. The conditioned medium (CM) were obtained and centrifuged (1000 rpm, $10 \mathrm{~min}$ ), and then the supernatant was filtered via a $0.22 \mu \mathrm{m}$ filter (Beyotime Biotechnology, China) and stored at $4{ }^{\circ} \mathrm{C}$ for treating the HUVECs. 


\section{Tube Formation Assay}

The tube formation assay was performed following the manufacturer's protocol (BD Biosciences, Franklin Lakes, NJ, https://www.bdbiosciences.com). $50 \mu \mathrm{L}$ growth factor reduced Matrigel (BD Biosciences company, San Jose, USA) was added to each well of a precooled 96-well plate and allowed to polymerize at $37^{\circ} \mathrm{C}$. Subsequently, the HUVECs or the HUVECs transfected (sh-ANG2/scramble ANG2) were treated with CM from SW480 cells (vector/WNT4-HA) and pretreated with ICG$001 \otimes 10 \mu \mathrm{M} \otimes 12$ hours before collection, and then cell suspension $(50 \mu \mathrm{L})$ at a concentration of $1 \times 10^{5}$ cells $/ \mathrm{mL}$ was paved onto the surface of matrix gel and incubated at $37^{\circ} \mathrm{C}$ for $12 \mathrm{~h}$. The cells were viewed under a microscope (OLYMPUS IX71, Japan) and photographed. Tube length was measured with ImageJ software (https://imagej.nih.gov/ij/, MD, USA).

\section{Suspended spheres formation}

The protocol used for analyzing sphere formation has been described previously[17]. Briefly, primary fibroblasts were labeled with PKH-26 (red) and mixed with GFP-transfected tumor cells (Scramble or sh-WNT4, WNT4-vector, or WNT4-HA) at a ratio of 3:1 in an ultra-low attachment plate (Corning, NY, USA) with DMEM medium at $37^{\circ} \mathrm{C}$ overnight. The typical heterospheroids were observed and counted using an inverted Olympus fluorescence microscope (OLYMPUS IX71).

\section{Collagen matrix contraction assay}

Contraction of collagen gels was performed in 96-well plates as previously reported [18]. Briefly, collagen gel (Corning, NY, USA), matrigel (Corning, NY, USA), FM, FBS, and suspended fibroblasts (with or without $400 \mathrm{ng} / \mathrm{mL}$ WNT4 pretreatment) were gently mixed. Then, $100 \mu \mathrm{l}$ of the mixture was added to each well in a 96-well plate and allowed to gel at $37^{\circ} \mathrm{C}$ for $30 \mathrm{~min}$ before the complete medium was added. For the inhibitory experiments, both gels and media were incubated with $10 \mu \mathrm{M}$ ICG001.

\section{Statistical analysis}

More than three times were repeated for all experiments. Data were presented as the mean \pm standard deviation (SD). The data of the relationships of WNT4, CA199, and CEA expression with the clinicopathological parameters of the CRC patients were presented as the mean \pm standard error of mean (SEM). The comparisons between two groups were performed by Student's t-tests or Mann-Whitney U tests for continuous variables. Survival probabilities and recurrence rates were estimated using the Kaplan-Meier method and differences between Kaplan-Meier curves were compared by using the log-rank test. All statistical tests were two-sided. SPSS v17.0 (Chicago, IL) was used to perform those statistical analyses. $P$ values $<0.05$ were considered statistically significant. ${ }^{*} P<0.05,{ }^{\star} * P<0.01$.

\section{Results}

\section{WNT4 was upregulated in serum and tissue of CRC patients}

We first analyzed the expression of WNT4 in CRC tissues and normal tissues from Oncomine Database, and found an elevated levels of WNT4 in CRC patients (Fig 1A, $P<0.05$ ). What's more, CRC patients with high WNT4 expression had lower survival rate and higher recurrence rate (Fig 1B, 1C, $P<0.05)$. In addition, tissues and serum samples were collected from CRC patients and healthy donors. ELISA was employed to measure the level of WNT4 in serum from 40 CRC patients and 28 healthy donors, significantly higher WNT4 levels was found in CRC than healthy donors (Fig 1D, $P<0.01)$. Interestingly, WNT4 level was decreased after radical surgery in $21 / 23$ (91.30\%) of CRC patients who accept surgery treatment to remove the tumor (Fig 1D, $P<0.05)$. Subsequently, the conditioned media from fresh tumor and adjacent normal tissues were collected to identify the source of WNT4 hypersecretion. The results showed that the WNT4 levels in conditioned medium from fresh tumors were significantly higher than those from adjacent tissues, which strongly identified WNT4 may be derived from CRC tissue (Fig 1E, $P<0.05$ ). This result may indicate that the aberrantly raised WNT4 in serum was secreted by CRC tumor tissues, which may also explain the decrease of WNT4 in serum after tumor resection. Also, those results suggested that the serum WNT4 level may be a potential biomarker for CRC. 
We next measured the expression of WNT4 in human CRC tissues and paired adjacent normal tissues by

immunohistochemistry (IHC). Compared with adjacent normal tissues, WNT4 was significantly increased in CRC (Fig 1F). IHC analysis of WNT4 in tumor tissue arrays indicated that the level of WNT4 was elevated in 45/63 (71.43\%) of tumors (Fig 1G). The expression of WNT4 in 4 paired of fresh frozen CRC tissues and adjacent normal tissues were evaluated by using western blot analysis, we found that WNT4 was upregulated in CRC tissue (Fig $1 \mathrm{H}, P<0.05)$.

These results suggested that WNT4 was upregulated in CRC serum and tissue, which may play a role in progression of CRC. Combined with the above finding that WNT4 level decreased after tumor resection, we could speculate that the aberrantly raised WNT4 in serum may be secreted by CRC tumor tissues.

\section{WNT4 level correlated with metastasis of CRC}

Next, we analyzed the relationships between the elevated WNT4 in serum and clinicopathological characteristics of CRC patients. As shown in Table 1, significantly different WNT4 expression were observed when comparing advanced late TNM T stage with early stage $(88.08 \mathrm{pg} / \mathrm{mL}$ vs. $55.94 \mathrm{pg} / \mathrm{mL} ; P=0.0293)$, and advanced TNM stage with early stage $(94.57 \mathrm{pg} / \mathrm{mL}$ vs. $66.35 \mathrm{pg} / \mathrm{mL} ; P=0.0464)$. In addition, higher levels of WNT4 were found in large tumor size $(96.60 \mathrm{pg} / \mathrm{mL}$ vs. 59.38 $\mathrm{pg} / \mathrm{mL} ; P=0.0087)$ and tumor with metastasis $(111.1 \mathrm{pg} / \mathrm{mL}$ vs. $71.73 \mathrm{pg} / \mathrm{mL} ; P=0.0428)$, while no relationship was observed between the WNT4 expression and age, sex, lymph node metastasis, and differentiation $(P>0.05)$. Remarkably, WNT4 showed a potential ability to differentiate different stages of CRC when compared with CA199 and CEA in our samples, for WNT4 levels were different at TNM stages and tumor size while CA199 and CEA were not (Table 1). These results indicated that high expression of WNT4 is associated with faster CRC enlarging and spreading, suggesting that WNT4 level correlated with metastasis of CRC.

\section{WNT4 promotes invasion and migration of CRC through $\beta$-catenin-dependent pathway in vitro}

Since we found that WNT4 is related to the clinical characteristics of CRC, we further explored the effect of WNT4 on CRC. Migration and invasion effects of WNT4 on CRC were measured when exogenous WNT4 added. Exogenous WNT4 at the concentration of $100 \mathrm{ng} / \mathrm{mL}$ greatly enhanced the migration and invasion ability of LoVo and HCT 116 cells (Fig 2A, 2B, $P<$ 0.05).

To further analyzed the mechanism of WNT4 promoting invasion and migration, TOP/FOP Flash assays were applied for testing the effect of WNT4 on Wnt/ $\beta$-catenin signaling pathway in CRC cells, as TOP/FOP Flash assays are a direct proof to test $\beta$-catenin-mediated transcriptional activity. E-cadherin and ZO-1 are two known markers involved in EMT were also measured $[19,20]$. The TCF/LEF transcription activity is significantly enhanced after treatment with WNT4 $(100 \mathrm{ng} / \mathrm{mL})$ for 2 h (Fig $2 \mathrm{C}, P<0.05)$, indicating that Wnt/ $\beta$-catenin signaling pathway was activated. Next, we found that the total $\beta$-catenin and AXIN2, a downstream target gene of $\beta$-catenin, were increased, while the levels of E-cadherin and ZO- 1 were decreased in LoVo and HCT 116 cells treated with exogenous WNT4 (100 ng/mL) for 0, 0.5, 1, 2, 6, and $24 \mathrm{~h}$, (Fig 2D, $P<0.05)$. What's more, the level of $\beta$-catenin in the nucleus also increased (Fig 2D, $P<0.05$ ), while AXIN2 was highest at one hour after treatment. The fluorescence microscopy also demonstrated that CRC cells treated with WNT4 raised $\beta$-catenin and AXIN2 accumulation and remarkably induced nuclear translocation of $\beta$-catenin and AXIN2 (Fig 2E). In order to further verify that WNT4 activates downstream pathways through canonical Wnt/ $\beta$-catenin pathways, an inhibitor of transcription mediated by $\beta$-catenin/TCF, ICG-001 was used. As shown in Fig 2F and 2G, $25 \mu \mathrm{M}$ ICG-001 completely inhibited activation of $\beta$-catenin signaling caused by WNT4 $(P<0.05)$.

In addition, CRC cells expressed different levels of WNT4 were also constructed. LoVo and HCT 116 highly expressed WNT4 while only low levels of WNT4 found in SW480. Therefore, LoVo, HCT 116 and SW480 were chosen to carry out the following related experiments. HCT 116 and LoVo cells transfected with WNT4-siRNA1, WNT4-siRNA2 and normal control siRNA \normal control $₫$ were constructed, respectively (Fig 3A, $P<0.05$ ). After validating the knockdown effects of WNT4-siRNAs in LoVo and HCT 116 cells, we successfully constructed stablely transfected WNT4-vector and WNT4-HA in SW480 cells (Fig 3B, $P<0.05)$. We found that the total $\beta$-catenin and AXIN2 in these cells decreased notably, while the levels of E-cadherin and ZO- 
1 increased in both loVo and HCT 116 cells transfected WNT4-siRNA1 and WNT4-siRNA2 (Fig 3C, $P<0.05$ ). Meanwhile, the level of $\beta$-catenin and AXIN2 in the nucleus also decreased (Fig $3 C, P<0.05$ ). Next, the TCF/LEF transcription activity in SW480 cells overexpressed WNT4 (WNT4-HA) significantly enhanced (Fig 3D, $P<0.05$ ). Those results may suggest that WNT4 promote migration and invasion of CRC through by promoting EMT, which through $\beta$-catenin-dependent pathway.

\section{WNT4 promotes invasion and migration of CRC through $\beta$-catenin-dependent pathway in vivo}

Next, to confirm our results in vitro, we then conducted a series of in vivo experiments. LoVo cells $\left(1 \times 10^{6}\right)$ stablely transfected with negative control shRNA (Scramble) or WNT4-shRNA were injected into the caudal vein of nude mice $(\mathrm{n}=7)$, respectively. Since liver metastasis is the most common distant metastasis of CRC, we checked the number of metastasis sites in the liver of those mice. Finally, liver metastasis occured in four mice for each group. The ratio of liver weight to body weight and the number of liver metastases in the Scramble group are much higher than the WNT4-shRNA group (Fig 3E, 3F, $P<0.05$ ). These results suggested that WNT4 may be a key player in promoting CRC metastasis.

To further explore if WNT4 had a pro-proliferation effect on CRC in vivo, a subcutaneous xenograft model using nude mice was constructed. Results showed that tumors developed from mice incubated with LoVo cells $\left(1 \times 10^{7}\right.$ cells $\left./ 200 \mu \mathrm{L}\right)$ transfected with WNT4-shRNA were smaller and lighter than that incubated with LoVo cells transfected with negative control shRNA (Scramble), though the difference is not statistically significant (Fig 3G, $P=0.0705, n=7$ ). In mice incubated with SW480 cells $\left(1 \times 10^{7}\right.$ cells $\left./ 200 \mu \mathrm{L}\right)$ stablely transfected WNT4-vector and WNT4-HA, tumors developed from WNT4-HA were larger and heavier than those developed from WNT4-vector cells, while no significant difference was observed (Fig $3 \mathrm{H}, P=0.1028, \mathrm{n}=5$ ). Those results may indicate that WNT4 only has a slight effect on tumor proliferation.

\section{WNT4 promotes fibroblast recruitment and activationvia $\beta$-catenin-dependent pathway}

Despite secretive WNT4 initiating EMT, as clearly illustrated above, the effect of WNT4 being highly secreted in the CRC microenvironment remains largely unknown. Activated Wnt/ $\beta$-catenin signaling has been implicated in fibrosis in a number of organs including the tumor stroma [21-23]. However, it is not clear whether WNT4 can also activate fibroblasts through $\beta$ catenin signaling. Strikingly, we found that a-SMA positive CAFs are more abundant in the samples from mice injected with the LoVo-Scramble $(n=7)$ and SW480-WNT4-HA-transfected cells $(n=5)$, compared to those from mice injected with the LoVoshWNT4 and SW480-WNT4-vectors, respectively (Fig 4A, 4B, $P=0.0005, P=0.0006$, respectively). Those results indicating that WNT4 may contribute to CRC tumor progression by recruiting and activating fibroblasts.

To investigate whether WNT4 can recruit fibroblasts, normal fibroblasts (NF) and cancer-associated fibroblasts (CAF) were isolated and identified from the tumor and adjacent tissues of CRC patients by an enzymatic dissociation method. The specific cell surface markers CD31, CD45, and CD329 were used to confirm the absence of endothelial, immune, and epithelial cell contamination by flow cytometry analysis (Supplementary Fig 1). We established a suspension coculture of CAFs and tumor cells with complete medium in ultra-low attachment plates. Intriguingly, fewer and smaller typical heterospheroids were formed in the WNT4-shRNA groups, supporting the hypothesis that WNT4 was related to the adhesive capacity of CRC cells and had the ability to recruit fibroblasts (Fig 4C, $P=0.0069$ ). Moreover, in the WNT4-HA group, more heterospheroids were found than WNT4-vector group, which further confirming our hypothesis (Fig 4D, $P<0.0001$ ).

In addition, exogenous WNT4 was added to the growth medium (without FBS) of normal fibroblasts (NF) at a final concentration of 0 (control) or $400 \mathrm{ng} / \mathrm{mL}$ for $24 \mathrm{~h}$. Stronger gel contraction was observed after the NF\#1 and NF\#2 treated with WNT4, indicating increased extracellular matrix remodeling (Fig 4E, $P=0.0139, P=0.00031$, respectively). We also tested the related markers of CAFs, fibronectin (FN) and a-SMA, by immunofluorescence and western blotting to verify the recruitment and contraction ability of WNT4 towards fibroblasts. Increased expression of FN and a-SMA were observed after the NFs were stimulated with WNT4 (400 ng, 24 h) (Fig 4F, 4G, 4H, $P<0.05)$. Together, these data highlight WNT4 as a novel and highly potent factor that could drive the conversion of NFs into CAFs within the tumor microenvironment. We next investigated the underlying mechanism of how WNT4 could activate NFs into CAFs. 
The WNT/ $\beta$-catenin pathway is known to play an important role in CRC-associated fibroblast [24, 25]. To interrogate the WNT4 signaling pathways operating in CAF activation, we first assessed the activation of the $\beta$-catenin-dependent pathway using immunofluorescence. As shown in Fig 5A, $\beta$-catenin nuclear translocation was observed in case of the NF\#1 and NF\#2 treated with WNT4 (400 ng/ml, $24 \mathrm{~h})$. These data suggest that WNT4 could activate the classical WNT/ $\beta$-catenin pathway in colorectal NFs. Subsequently, ICG-001 was used to block the canonical $\beta$-caten in pathway, we found that ICG-001 completely blocked elevation of a-SMA and FN at a concentration of $10 \mu \mathrm{M}$ (Fig 5B, 5C, $P<0.05$ ). Additionally, the effects of the WNT4 protein on heterospheroid formation and gel contraction ability were also completely inhibited by ICG-001 (Fig 5D and 5E, $P<$ 0.05).

\section{WNT4 promote angiogenesis in CRC via a WNT4/ß-catenin/ANG2 pathway}

Angiogenesis is very important in the development of tumor, therefore, we next studied whether WNT4 could promote angiogenesis in CRC. In subcutaneous xenograft mouse model, we found higher abundance of endothelial (CD31) were found in tumors from mice incubated with LoVo cells transfected Scramble and SW480 cells transfected WNT4-HA than that from mice incubated with LoVo cells transfected WNT4-shRNA and SW480 cells transfected WNT4-vector, respectively (Fig 6A, 6B, $P<0.05)$. These results indicate that WNT4 may promote angiogenesis in CRC, while the detailed mechanism need to be elucidated.

To investigate the possible role of WNT4 in angiogenesis, HUVECs were treated with CMs from CRC cells with different WNT4 expression levels, and effects of WNT4 on the ability in generating tubular networks of HUVECs on Matrigel was evaluated. Our results showed that the tube formation was significantly promoted when the HUVECs treated with CM from SW480 transfected with WNT4-HA than that from SW480 transfected WNT4-vector (Fig 6C, $P<0.05$ ). These results indicate that angiogenesis could be promoted by WNT4.

To explore whether WNT4 promotes tumor angiogenesis via $\beta$-catenin pathway, TOP/FOP Flash assay was used to detect TCF/LEF transcription activity when HUVECs treated with CM from SW480 cells with different levels of WNT4 (as described above). HUVECs treated with CRC cells with high WNT4 expression resulted in upregulation of transcriptional activity (Fig 6D, $P<0$.05). Subsequently, we found that $\beta$-catenin transcriptional activity was downregulated, and tube formation was obviously abrogated after blocking by ICG-001 (10 $\mathrm{MM})$ (Fig 6C, 6D, $P<0.05)$. Those results demonstrated that WNT4 could promote angiogenesis via a $\beta$-catenin-dependent pathway.

We next measured the expression of ANG2 to investigate whether ANG2 could be regulated by WNT4/ $\beta$-catenin pathway. RTqPCR and ELISA were used to measure the expression of ANG2 from different treated HUVECs (Fig 6E, 6F, $P<0.05)$. We can infer that ANG2 was upregulated with high levels of WNT4, while it was downregulated after blocking by ICG-001 (10 $\mu$ M) (Fig $6 \mathrm{E}, 6 \mathrm{~F}, P<0.05)$. In addition, HUVECs stablely transfected with negative control shRNA (Scramble) and sh-ANG2 were constructed and the expression of ANG2 were identified by RT-qPCR and ELISA (Fig 6G, 6H, $P<0.01$ ). The tube formation promoted by high WNT4 expression were significantly inhibited due to low ANG2 expression (Fig $6 \mathbf{l}, P<0.01$ ). Those results could indicate that angiogenesis could be regulated by WNT4/ $\beta$-catenin/ANG2 cascade.

\section{WNT4 levels could be regulated by miR-497}

MiR-497 has been well characterized as a tumor suppressor in previous work by our group [26]. For further study, we found that WNT4 expression were decreased at both mRNA and proteins levels in LoVo and HCT 116 CRC cell lines after transfecting with miR-497 mimics (Fig 7A, $P<0.05$ ). To further illustrate the relationship between miR-497 and WNT4, a DualLuciferase Reporter Assay was conducted (Fig 7B). It was also found that miR-497 was able to suppress the reporter gene activity of WNT4 in LoVo cells (Fig 7C, $P<0.05$ ), whereas mutant plasmids showed no change in the reporter gene activity (Fig 7C, $P>0.05)$. Next, the expression of miR-497 and WNT4 among 10 randomly selected CRC tissues and paired adjacent tissues were investigated by quantitative real-time PCR (qRT-PCR). Significant downregulation of miR-497 and upregulation of WNT4 expression were found (Fig 7D, 7E). Moreover, a negative correlation was found between miR-497 and the WNT4 expression 
levels was also seen in those CRC cancer tissues and paired adjacent tissues (Fig 7F, $P<0.01$ ). These data suggested that miR497 could downregulate the level of WNT4.

\section{Discussion}

WNT ligands are frequently reported to play an important role in CRC, but the role of WNT4 on CRC was rarely studied. It has been reported WNT4 promoted proliferation of breast cancer stem cells [27], and promote gastric cancer [28], suggesting an pro-carcinogenic function of WNT4. To our knowledge, the levels of WNT4 in serum has not been identified previously. In the present study, for the first time, we demonstrated that WNT4 was elevated in CRC patient serum and originated from tumor tissues, while it was decreased after tumor resection, indicating that WNT4 may be a potential biomarker for CRC. Besides, analysis of relationships between WNT4 in serum and clinicopathological characteristics showed that elevated WNT4 correlated with advanced stage and metastasis of CRC. The detection of tumor-associated biomarkers in peripheral blood of cancer patients provides an opportunity to analyze the changes in tumor burden and monitor the responses to treatment. The results in this study suggest that WNT4 could potentially be a serum marker for the diagnosis, and value the risk of metastasis for CRC.

Moreover, compared to CEA and CA199 levels in serum, we found that WNT4 in serum had significantly higher sensitivity and specificity. However, due to the lack of a robustly designed prospective trial, this result remains subject to further verification. Taken together, our results highlight the potential clinical significance of WNT4, suggesting that further studies are needed to investigate the diagnostic applications of WNT4 in CRC

Although the upregulation of WNT expression had been extensively investigated in CRC and corresponding liver metastasis [29-31], the role of WNT4 in CRC has not been explored. In our study, we identified WNT4 could promote metastasis of CRC through WNT4/ $\beta$-catenin cascade via EMT. Cancer cells often required mesenchymal phenotype to enhance their ability of invasion and metastasis [32], which is consistent with our study that WNT4 could promote EMT in CRC cells to promote invasion and migration in vitro and in vivo. What's more, most studies focused on WNT's role in cancer cells, Wnt/ $\beta$-Catenin pathway, for example, could promotes gastric cancer cells [33], non-small cell lung cancer [34] and colorectal cancer cells [35], while little attention paid to its possible role in the tumor microenvironment, including CAFs and endothelial cells. In this paper, we showed that WNT4 could activated surrounding fibroblasts through WNT4/ $\beta$-catenin pathway. Furthermore, recent evidence suggests a significant role for EMT in fibrosis, including its involvement in tumor stroma activation [24, 36]. In our current study, WNT4 prompted the nuclear translocation of $\beta$-catenin and increased the expression of a-SMA and fibronectin in NFs, which subsequently enhanced reinforced cell contraction and microsphere formation. ANG2 was a critical gene for angiogenesis, while the correlation of ANG2 and WNT4/ $\beta$-catenin has never been investigated before. In our study, we identified that angiogenesis could be activated by WNT4 through WNT4/ß-catenin/ANG2 pathway, which is vital for tumor invasion and metastasis. As far as we know, few studies have explored the effect of WNT4 on those components in CRC microenvironment. However, our deficiency lies in the lack of deep research on the mechanisms.

It is also worth noting that we found those effects of WNT4 at the concentration that reported in previous studies [37, 38], while no effect was found at serum concentration. Combined with our results, we speculated that WNT4 secreted by colorectal cancer tissue could mainly acts on the tumor microenvironment to create an environment that is conducive to its growth, while WNT4 in serum may simply act as a diagnostic or prognostic indicator for CRC.

In addition, we also found that downregulated miR-497 may lead to elevated WNT4 in CRC tissues. Downregulation of miR497 and its tumor-suppressive role have been reported in multiple cancers [39-42]. Our work showed WNT4 was a novel direct target of miR-497. However, further explorations are needed to confirm the regulatory effect of miR-497 on WNT4 expression and role in the progression of CRC.

\section{Conclusion}

Page $11 / 22$ 
In our work, we demonstrated for the first time that WNT4 could be a potential biomarker for CRC, which could be used to diagnose and value the risk of metastasis for CRC. Our data then substantiate the effects of secretory WNT4 on the development of the colorectal tumor microenvironment. WNT4 promoted CRC progression and liver metastasis through the activation of WNT4/ $\beta$-catenin signaling. EMT could be induced by WNT4 to promote the migration of CRC cells via WNT4/ $\beta$ catenin signaling. Moreover, fibroblasts were also activated by WNT4 through WNT4/ $\beta$-catenin signaling and transformed from NFs into CAFs, which increases the number of CAFs in the tumor stroma and further promoted the metastasis of CRC cells. Moreover, angiogenesis induced by WNT4/ $\beta$-catenin/ANG2 signaling could also promote metastasis of CRC. In conclusion, the present study has revealed that WNT4 was a novel molecule involved in CRC progression, which may provide novel clues for the diagnosis and treatment of CRC.

\section{Abbreviations}

BSA, bovine serum albumin; CRC, Colorectal cancer; CAF, cancer associated fibroblast; EMT, epithelial-to-mesenchymal transition; IHC, immunohistochemical staining; NF, normal fibroblast; shRNA, short hairpin RNA; siRNA, small Interference RNA; RT-qPCR, real-time quantitative polymerase chain reaction.

\section{Declarations}

\section{Availability of data and materials}

All remaining data are available within the article and supplementary files, or available from the authors upon request.

\section{Ethics approval and consent to participate}

The study was authorized by the Ethic Committee of the Renmin Hospital of Wuhan University. Written informed consent was provided from each subject. The procedures in this study were permitted by the Animal Research Committee of the Renmin Hospital of Wuhan University.

\section{Consent for publication}

All authors have agreed to publish this manuscript.

\section{Competing interests}

No potential conflicts of interest were disclosed.

\section{Fundings}

This work was supported by the National Natural Science Foundation of China (grant number 81672387 and 81770899 ).

\section{Author contributions}

DMY, QL, LWY, LLW, MJZ: conceptualization and data analysis; LHZ, MX, RDS, ZHL, JZ, LH, XDH, DC: Investigation and methodology; DMY, QL, RDS: validation and visualization; HGY and YNY: project administration, funding acquisition, supervision; HGY, YNY, DMY, QL, RDS: manuscript writing and revision.

\section{Acknowledgments}

We thank Ms. Ying Wang for excellent technical assistance in this work. 


\section{References}

1. Torre LA, Bray F, Siegel RL, Ferlay J, Lortet-Tieulent J, Jemal A. Global cancer statistics, 2012. CA Cancer J Clin. 2015;65(2):87-108.

2. Cullinane CM, Creavin B, O'Connell EP, Kelly L, O'Sullivan MJ, Corrigan MA, et al., Risk of colorectal cancer associated with BRCA1 and/or BRCA2 mutation carriers: systematic review and meta-analysis. Br J Surg, 2020.

3. Bray F, Ferlay J, Soerjomataram I, Siegel RL, Torre LA, Jemal A. Global cancer statistics 2018: GLOBOCAN estimates of incidence and mortality worldwide for 36 cancers in 185 countries. CA Cancer J Clin. 2018;68(6):394-424.

4. Sparano JA, Gray RJ, Makower DF, Pritchard KI, Albain KS, Hayes DF, et al. Adjuvant Chemotherapy Guided by a 21-Gene Expression Assay in Breast Cancer. N Engl J Med. 2018;379(2):111-21.

5. Periyakaruppan A, Gandhiraman RP, Meyyappan M, Koehne JE. Label-free detection of cardiac troponin-I using carbon nanofiber based nanoelectrode arrays. Anal Chem. 2013;85(8):3858-63.

6. Zhang B, Wang M, Gong A, Zhang X, Wu X, Zhu Y, et al. HucMSC-Exosome Mediated-Wnt4 Signaling Is Required for Cutaneous Wound Healing. Stem Cells. 2015;33(7):2158-68.

7. Clevers H, Nusse R. Wnt/beta-catenin signaling and disease. Cell. 2012;149(6):1192-205.

8. Gascard P, TIsty TD. Carcinoma-associated fibroblasts: orchestrating the composition of malignancy. Genes Dev. 2016;30(9):1002.

9. Kalluri R, Zeisberg M. Fibroblasts in cancer. Nat Rev Cancer. 2006;6(5):392-401.

10. He XJ, Tao HQ, Hu ZM, Ma YY, Xu J, Wang HJ, et al. Expression of galectin-1 in carcinoma-associated fibroblasts promotes gastric cancer cell invasion through upregulation of integrin beta1. Cancer Sci. 2014;105(11):1402-10.

11. Vered M, Dayan D, Yahalom R, Dobriyan A, Barshack I, Bello IO, et al. Cancer-associated fibroblasts and epithelialmesenchymal transition in metastatic oral tongue squamous cell carcinoma. Int J Cancer. 2010;127(6):1356-62.

12. Heichler C, Scheibe K, Schmied A, Geppert Cl, Schmid B, Wirtz S, et al., STAT3 activation through IL-6/IL-11 in cancerassociated fibroblasts promotes colorectal tumour development and correlates with poor prognosis. Gut, 2019.

13. Lee KW, Yeo SY, Sung CO, Kim SH. Twist1 is a key regulator of cancer-associated fibroblasts. Cancer Res. 2015;75(1):7385.

14. Orso F, Quirico L, Dettori D, Coppo R, Virga F, Ferreira LC, et al. Role of miRNAs in tumor and endothelial cell interactions during tumor progression. Semin Cancer Biol. 2020;60:214-24.

15. Zhang B, Wu X, Zhang X, Sun Y, Yan Y, Shi H, et al. Human umbilical cord mesenchymal stem cell exosomes enhance angiogenesis through the Wnt4/beta-catenin pathway. Stem Cells Transl Med. 2015;4(5):513-22.

16. Ji C, Yue S, Gu J, Kong Y, Chen H, Yu C, et al. 2,7-Dibromocarbazole interferes with tube formation in HUVECs by altering Ang2 promoter DNA methylation status. Sci Total Environ. 2019;697:134156.

17. Gao Q, Yang Z, Xu S, Li X, Yang X, Jin P, et al. Heterotypic CAF-tumor spheroids promote early peritoneal metastatis of ovarian cancer. J Exp Med. 2019;216(3):688-703.

18. Avgustinova A, Iravani M, Robertson D, Fearns A, Gao Q, Klingbeil P, et al. Tumour cell-derived Wnt7a recruits and activates fibroblasts to promote tumour aggressiveness. Nat Commun. 2016;7:10305.

19. Stump RJ, Lovicu FJ, Ang SL, Pandey SK, McAvoy JW. Lithium stabilizes the polarized lens epithelial phenotype and inhibits proliferation, migration, and epithelial mesenchymal transition. J Pathol. 2006;210(2):249-57.

20. Zhong Q, Zhou B, Ann DK, Minoo P, Liu Y, Banfalvi A, et al. Role of endoplasmic reticulum stress in epithelialmesenchymal transition of alveolar epithelial cells: effects of misfolded surfactant protein. Am J Respir Cell Mol Biol. 2011;45(3):498-509.

21. Zhou D, Fu H, Zhang L, Zhang K, Min Y, Xiao L, et al., Tubule-Derived Wnts Are Required for Fibroblast Activation and Kidney Fibrosis. Journal of the American Society of Nephrology Jasn, 2017. 28(8): p. ASN.2016080902. 
22. Xiang FL, Fang M, Yutzey KE. Loss of $\beta$-catenin in resident cardiac fibroblasts attenuates fibrosis induced by pressure overload in mice. Nat Commun. 2017;8(1):712.

23. Xu L, Cui WH, Zhou WC, Li DL, Li LC, Zhao P, et al. Activation of Wnt/beta-catenin signalling is required for TGFbeta/Smad2/3 signalling during myofibroblast proliferation. J Cell Mol Med. 2017;21(8):1545-54.

24. Niell N, Larriba MJ, Ferrer-Mayorga G, Sã n-Prl, Cantero R, Real FX, et al., The human PKP2/Plakophilin-2 gene is induced by Wnt/Îl2-catenin in normal and colon cancer-associated fibroblasts. International Journal of Cancer, 2017. 142(4).

25. Hu YB, Yan C, Mu L, Mi YL, Zhao H, Hu H, et al., Exosomal Wnt-induced dedifferentiation of colorectal cancer cells contributes to chemotherapy resistance. Oncogene, 2018.

26. Ding Q, He K, Luo T, Deng Y, Wang H, Liu H, et al. SSRP1 Contributes to the Malignancy of Hepatocellular Carcinoma and Is Negatively Regulated by miR-497. Mol Ther. 2016;24(5):903-14.

27. Brisken C, Hess K, Jeitziner R. Progesterone and Overlooked Endocrine Pathways in Breast Cancer Pathogenesis. Endocrinology. 2015;156(10):3442-50.

28. Zhu Y, Zhang B, Gong A, Fu H, Zhang X, Shi H, et al. Anti-cancer drug 3,3'-diindolylmethane activates Wnt4 signaling to enhance gastric cancer cell stemness and tumorigenesis. Oncotarget. 2016;7(13):16311-24.

29. Lee MA, Park JH, Si YR, Oh ST, Kang WK, Kim HN. Wnt3a expression is associated with MMP-9 expression in primary tumor and metastatic site in recurrent or stage IV colorectal cancer. Bmc Cancer. 2014;14(1):125-5.

30. Qi L, Sun B, Liu Z, Cheng R, Li Y, Zhao X. Wnt3a expression is associated with epithelial-mesenchymal transition and promotes colon cancer progression. J Exp Clin Cancer Res. 2014;33(1):107.

31. Zhang W, Sun Z, Su L, Wang F, Jiang Y, Yu D, et al. miRNA-185 serves as a prognostic factor and suppresses migration and invasion through Wnt1 in colon cancer. Eur J Pharmacol. 2018;825:75-84.

32. Chaffer CL, San Juan BP, Lim E, Weinberg RA. EMT, cell plasticity and metastasis. Cancer Metastasis Rev. 2016;35(4):645-54.

33. Cong N, Du P, Zhang A, Shen F, Su J, Pu P, et al. Downregulated microRNA-200a promotes EMT and tumor growth through the wnt/beta-catenin pathway by targeting the E-cadherin repressors ZEB1/ZEB2 in gastric adenocarcinoma. Oncol Rep. 2013;29(4):1579-87.

34. Yang S, Liu Y, Li MY, Ng CSH, Yang SL, Wang S, et al. FOXP3 promotes tumor growth and metastasis by activating Wnt/beta-catenin signaling pathway and EMT in non-small cell lung cancer. Mol Cancer. 2017;16(1):124.

35. Hu TH, Yao Y, Yu S, Han LL, Wang WJ, Guo H, et al. SDF-1/CXCR4 promotes epithelial-mesenchymal transition and progression of colorectal cancer by activation of the Wnt/beta-catenin signaling pathway. Cancer Lett. 2014;354(2):41726.

36. Kramer N, Schmollerl J, Unger C, Nivarthi H, Rudisch A, Unterleuthner D, et al. Autocrine WNT2 signaling in fibroblasts promotes colorectal cancer progression. Oncogene. 2017;36(39):5460-72.

37. Bader E, Migliorini A, Gegg M, Moruzzi N, Gerdes J, Roscioni SS, et al. Identification of proliferative and mature beta-cells in the islets of Langerhans. Nature. 2016;535(7612):430-4.

38. Yucer N, Holzapfel M, Jenkins Vogel T, Lenaeus L, Ornelas L, Laury A, et al. Directed Differentiation of Human Induced Pluripotent Stem Cells into Fallopian Tube Epithelium. Sci Rep. 2017;7(1):10741.

39. Zhiyuan H, Yanbin Z, Qiaoyuan Y, Binbin L, Jianjun W, Yajie Z, et al. miR-497 and miR-34a retard lung cancer growth by co-inhibiting cyclin E1 (CCNE1). Oncotarget. 2015;6(15):13149-63.

40. Xu JW, Wang TX, You L, Zheng LF, Shu H, Zhang TP, et al. Insulin-like growth factor 1 receptor (IGF-1R) as a target of MiR497 and plasma IGF-1R levels associated with TNM stage of pancreatic cancer. PLoS One. 2014;9(3):e92847.

41. Shaohua X, Guang-Bo F, Zhen T, Jun OY, Fanfei K, Bing-Hua J, et al. MiR-497 decreases cisplatin resistance in ovarian cancer cells by targeting mTOR/P70S6K1. Oncotarget. 2015;6(28):26457-71.

42. Xiang-Jie K, Liu-Jian D, Xiao-Qiang Q, Ding X, Hai-Long L, Ying-Jian Z, et al. Tumor-suppressive microRNA-497 targets IKK $\beta$ to regulate NF-KB signaling pathway in human prostate cancer cells. American Journal of Cancer Research.

Page $14 / 22$ 


\section{Tables}

Table1. Relation of WNT4, CA199, CEA expression with clinicopathological parameters of patients with CRC.

\begin{tabular}{|c|c|c|c|c|c|c|c|c|c|}
\hline \multirow[t]{2}{*}{ Parameters } & \multicolumn{3}{|c|}{ WNT4 (pg/mL) } & \multicolumn{3}{|c|}{ CA199 (U/mL) } & \multicolumn{3}{|c|}{ CEA(ng/mL) } \\
\hline & Cases & Mean \pm SEM & $\begin{array}{l}P \\
\text { value }\end{array}$ & Cases & Mean \pm SEM & $\begin{array}{l}P \\
\text { value }\end{array}$ & Cases & Mean \pm SEM & $\begin{array}{l}P \\
\text { value }\end{array}$ \\
\hline \multicolumn{10}{|l|}{ Age(y) } \\
\hline$\leq 65$ & 23 & $77.31 \pm 10.04$ & 0.9583 & 22 & $31.51 \pm 10.27$ & 0.3766 & 22 & $4.304 \pm 1.315$ & 0.7307 \\
\hline$>65$ & 17 & $78.07 \pm 9.623$ & & 16 & $19.78 \pm 5.963$ & & 16 & $3.671 \pm 1.143$ & \\
\hline \multicolumn{10}{|l|}{ Sex } \\
\hline Male & 20 & $88.46 \pm 11.14$ & 0.1129 & 19 & $28.36 \pm 9.941$ & 0.7862 & 19 & $4.445 \pm 1.394$ & 0.6533 \\
\hline Female & 20 & $66.81 \pm 7.998$ & & 19 & $24.79 \pm 8.484$ & & 19 & $3.629 \pm 1.141$ & \\
\hline \multicolumn{10}{|l|}{ TNM T } \\
\hline$I \sim \|$ & 13 & $55.94 \pm 8.395$ & 0.0293 & 13 & $26.84 \pm 12.39$ & 0.9768 & 12 & $1.135 \pm 0.237$ & 0.0247 \\
\hline $\mathrm{III} \sim \mathrm{IV}$ & 27 & $88.08 \pm 8.941$ & & 25 & $26.44 \pm 7.594$ & & 26 & $5.377 \pm 1.216$ & \\
\hline \multicolumn{10}{|l|}{ TNM N } \\
\hline 0 & 26 & $73.99 \pm 9.211$ & 0.4849 & 26 & $28.20 \pm 8.763$ & 0.7158 & 25 & $2.732 \pm 0.769$ & 0.0403 \\
\hline $1-2$ & 14 & $84.39 \pm 10.49$ & & 12 & $23.05 \pm 7.982$ & & 13 & $6.548 \pm 2.021$ & \\
\hline \multicolumn{10}{|l|}{ TNM M } \\
\hline 0 & 34 & $71.73 \pm 6.868$ & 0.0428 & 32 & $21.28 \pm 5.175$ & 0.0570 & 32 & $3.562 \pm 0.76$ & 0.2221 \\
\hline 1 & 6 & $111.1 \pm 22.62$ & & 6 & $54.80 \pm 29.61$ & & 6 & $6.575 \pm 4.071$ & \\
\hline \multicolumn{10}{|l|}{ TNM stage } \\
\hline$I \sim \|$ & 24 & $66.35 \pm 8.097$ & 0.0464 & 24 & $22.71 \pm 6.797$ & 0.4407 & 23 & $2.710 \pm 0.836$ & 0.0642 \\
\hline $\mathrm{III} \sim \mathrm{IV}$ & 16 & $94.57 \pm 11.61$ & & 14 & $33.20 \pm 13.27$ & & 15 & $6.072 \pm 1.772$ & \\
\hline \multicolumn{10}{|l|}{ Grade } \\
\hline WD & 8 & $62.62 \pm 14.33$ & 0.5367 & 8 & $37.70 \pm 19.55$ & 0.6844 & 8 & $1.52 \pm 0.41$ & 0.3173 \\
\hline MD & 28 & $82.48 \pm 8.83$ & & 27 & $23.70 \pm 7.14$ & & 26 & $4.89 \pm 1.24$ & \\
\hline PD & 4 & $73.76 \pm 16.26$ & & 3 & $22.84 \pm 3.32$ & & 4 & $3.50 \pm 1.59$ & \\
\hline \multicolumn{10}{|l|}{ Tumor size } \\
\hline$\leqslant 3 \mathrm{~cm}$ & 18 & $59.38 \pm 8.190$ & 0.0087 & 17 & $25.53 \pm 9.579$ & 0.8158 & 17 & $3.853 \pm 1.725$ & 0.7530 \\
\hline$>3 \mathrm{~cm}$ & 20 & $96.60 \pm 10.38$ & & 19 & $28.77 \pm 9.850$ & & 19 & & \\
\hline
\end{tabular}

WD, Well differentiated; MD, Moderately differentiated; PD, Poorly differentiated. Statistical significance was defined as $P<$ 0.05 . 


\section{A}

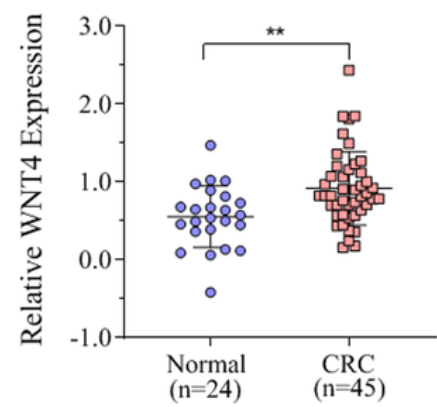

D

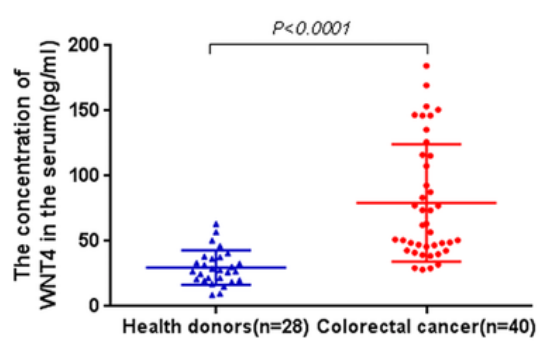

B
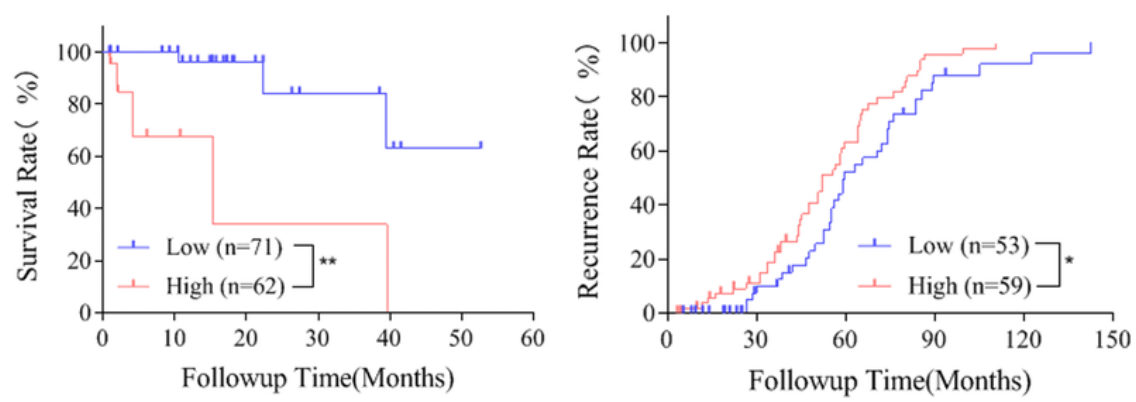

$\mathbf{E}$
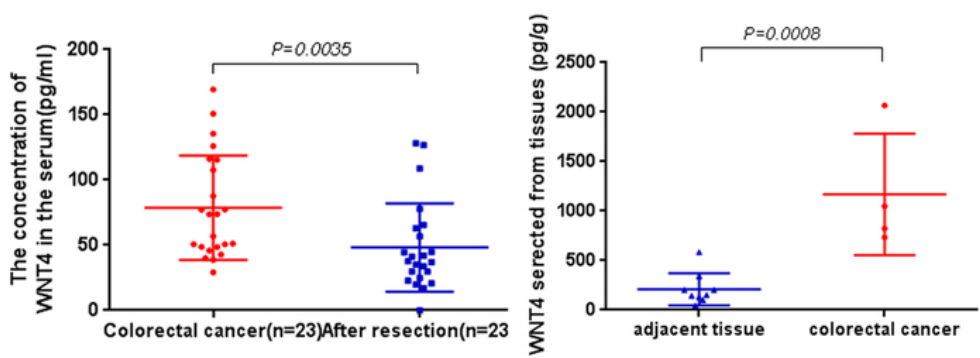

${ }_{40 x}$
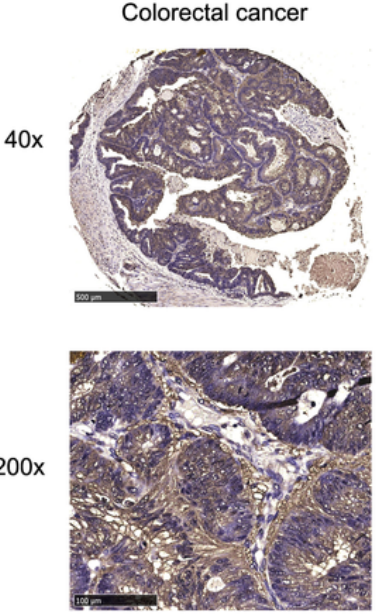

Adjacent normal mucosa
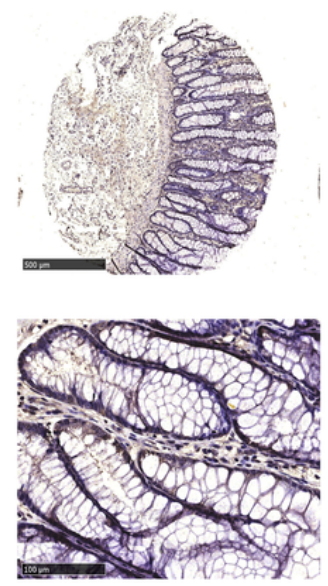

G

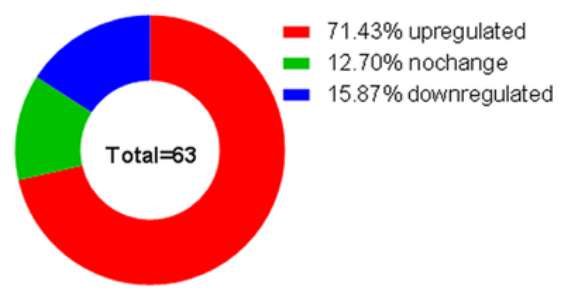

\section{H}

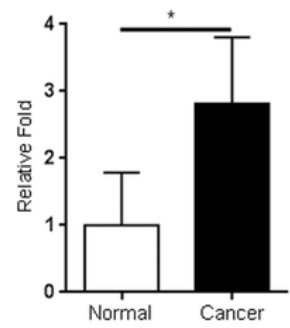

Figure 1

WNT4 was upregulated in CRC. (A) WNT4 expression level in CRC tissue $(n=45)$ and normal tissue $(n=24)$ from the Oncomine Database. (B and C) CRC patients with higher WNT4 expression had lower survival rate and higher recurrence rate from The Cancer Genome Atlas Colorectal (low WNT4 group, n=71; high WNT4 group, $n=62$ ) and Jorissen Colorectal 3 (low WNT4 group, $n=53$; high WNT4 group, $n=59$ ) respectively. (D) The WNT4 level in the serum in the CRC patients were higher than that in healthy donors, while it was decreased after radical surgery in 21/23 (91.30\%) of CRC patients. (E) WNT4 levels secreted from per gram of CRC tissues and adjacent tissue after overnight culture. (F, G and H) The expression of WNT4 was significantly higher in colorectal cancer tissue compared to the adjacent tissue in IHC and western blots. Data are presented as the mean \pm SD. Two-tailed Student's $t$ test was used for statistical analyses. ${ }^{*} P<0.05,{ }^{*} P<0.01$. 

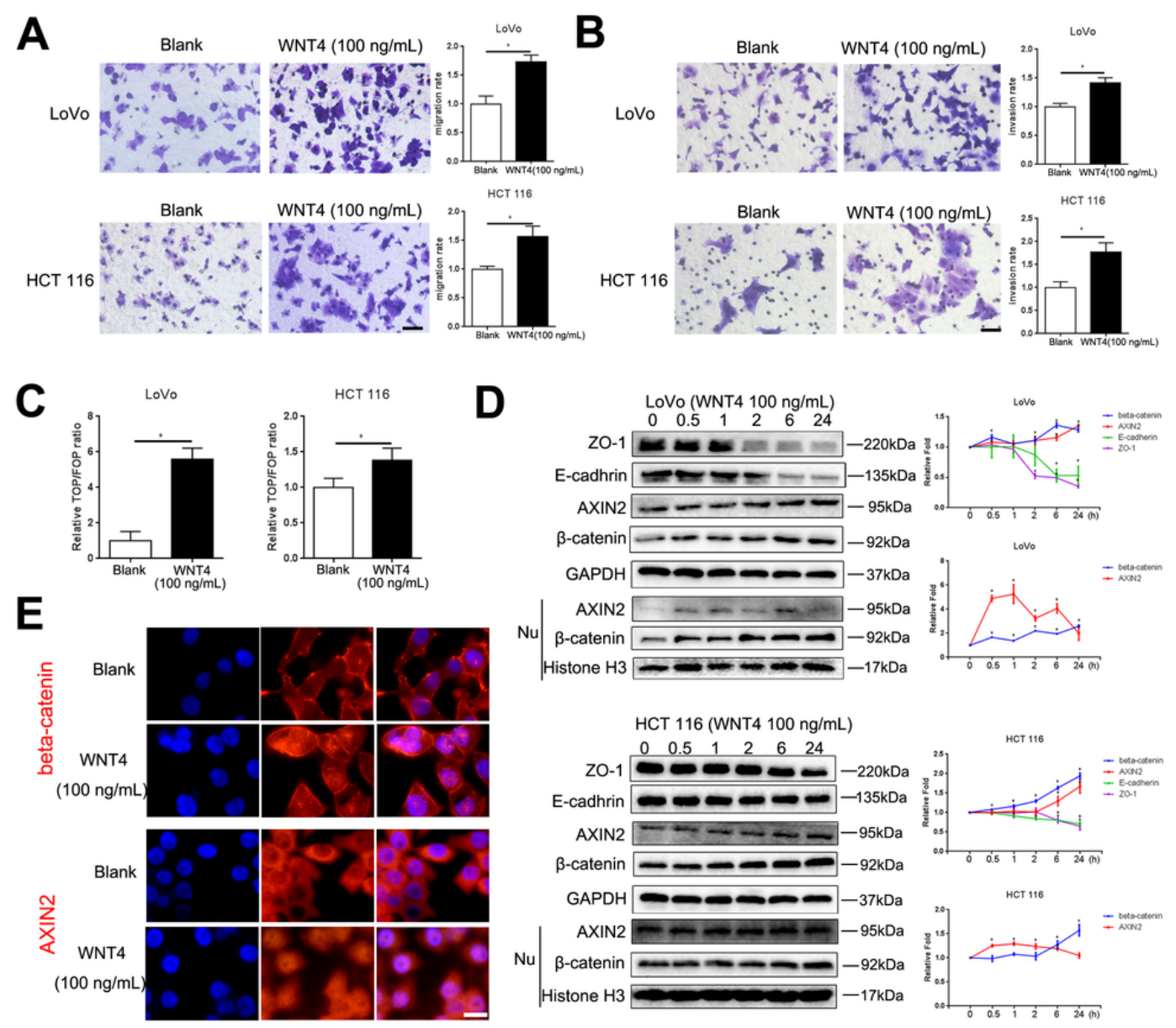

$\mathbf{F}$
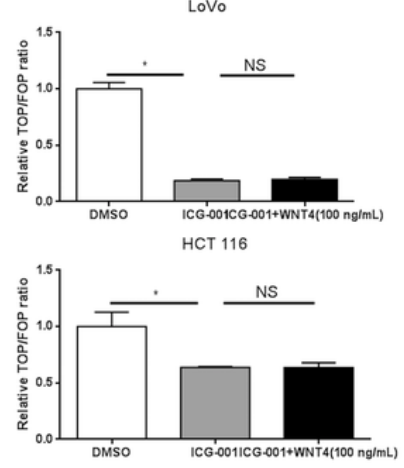

G
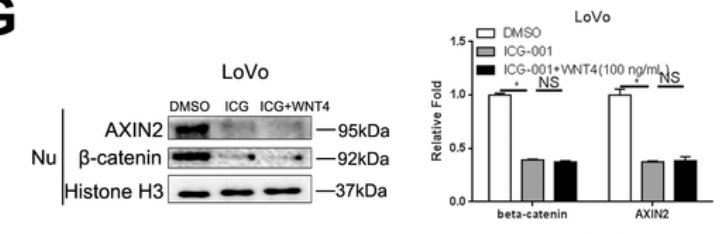

15 믐 0 MSO
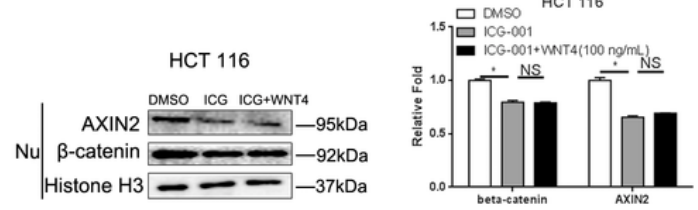

Figure 2

WNT4 induced the expression of $\beta$-catenin. (A and B) Cell migration and invasion capabilities of CRC cells pretreated with WNT4 $(100 \mathrm{ng} / \mathrm{mL})$ for 24 hours. Scale bar, $50 \mu \mathrm{m}$. (C) TOP-flash plasmid or FOP-flash $(2 \mathrm{mg})$ was transfected to the CRC cells for $48 \mathrm{~h}$; the cells were treated with WNT4 $(100 \mathrm{ng} / \mathrm{mL})$ for 6 hours before analysis. (D) LoVo and HCT 116 CRC cells were treated with WNT4 (100 ng/mL); and expression of $\beta$-catenin, AXIN2, E-cadherin, ZO-1, and nuclear $\beta$-catenin and AXIN2 were determined by western blots. (E) Higher expression and nuclear translocalization of $\beta$-catenin and AXIN2 were shown in immunofluorescence after LoVo cells treated with WNT4 $(100 \mathrm{ng} / \mathrm{mL})$ for 6 hours. Scale bar, $10 \mu \mathrm{m}$. (F and G) TOP/FOP-Flash reporter assay and western blots were used to detect whether the WNT pathway is activated. CRC cells were treated with ICG$001(25 \mu \mathrm{M})$ for 24 hours and WNT4 $(100 \mathrm{ng} / \mathrm{mL})$ for 6 hours. Two-tailed Student's t test and ANOVA were used for statistical analyses. All experiments were performed in triplicate. Measurement data were presented as the mean $\pm S D$. *P $<0.05 ; N S$, no significance. 
A
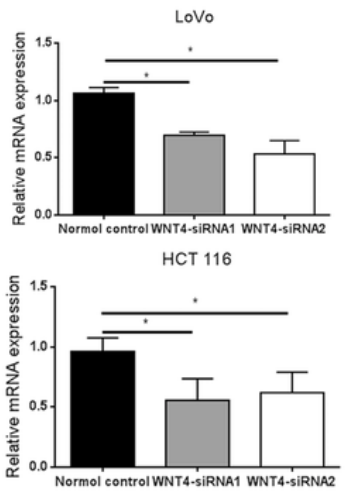

B

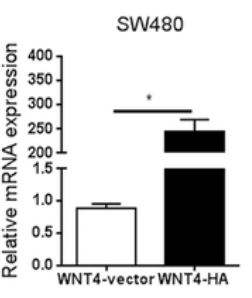

D
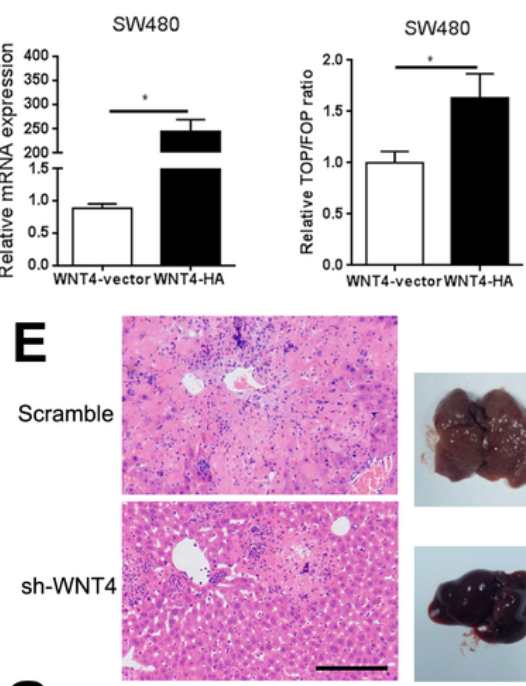

G

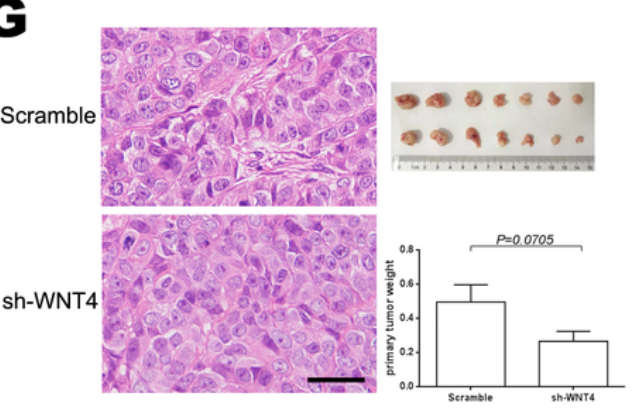

C
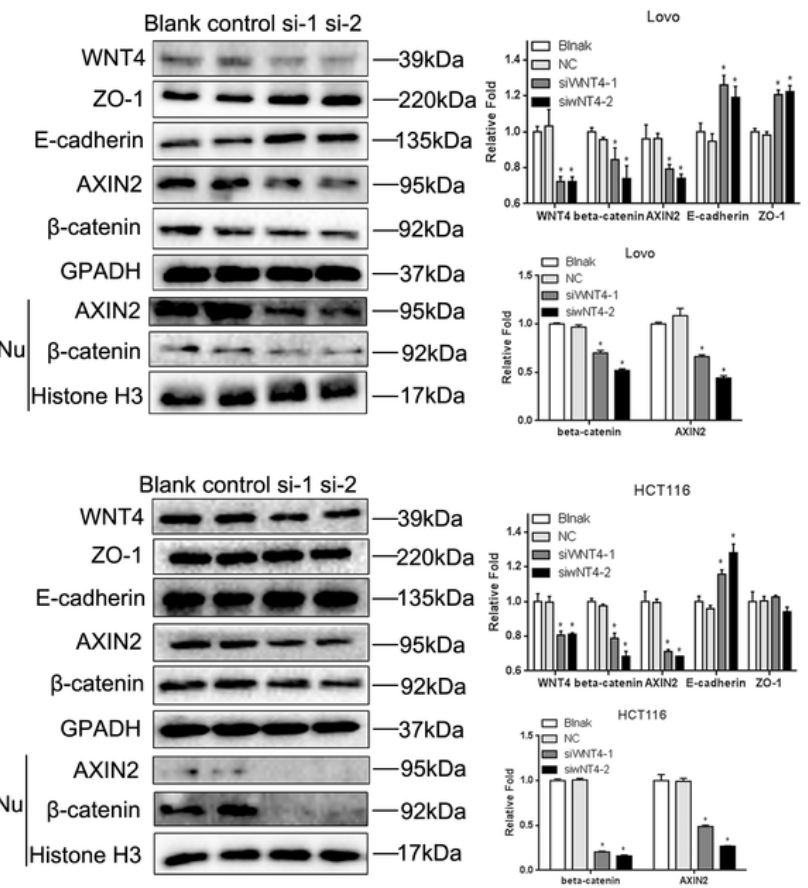

F
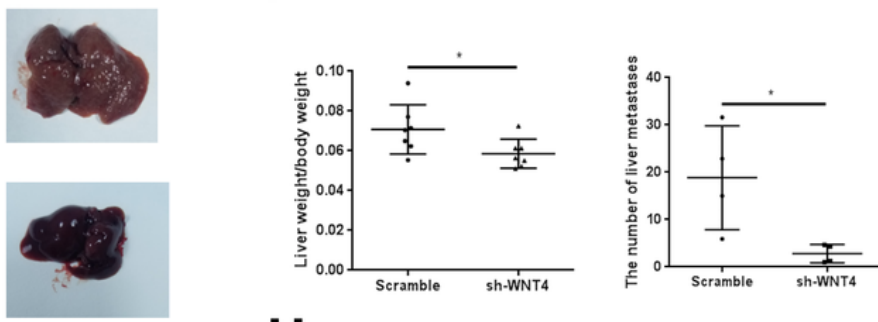

H

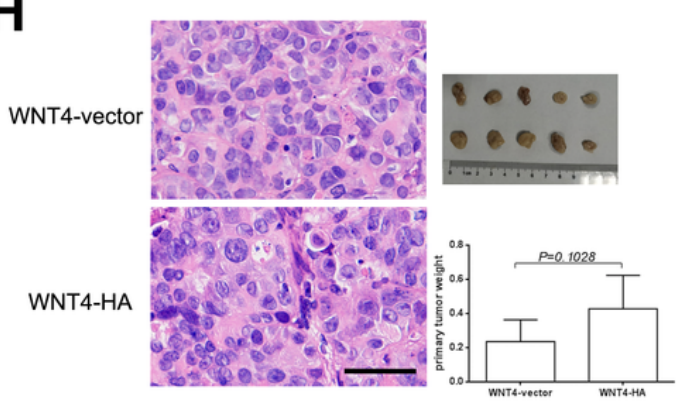

Figure 3

WNT4 can promote metastasis of colorectal cancer cells. (A) The mRNA level of WNT4 in CRC cells transfected with WNT4siRNA1, WNT4-siRNA2, and normal control. (B) The mRNA level of WNT4 in SW480 cells transfected with WNT4-vector and WNT4-HA. (C) CRC cells were transfected with WNT4-siRNA1, WNT4-siRNA2, and then, WNT4, $\beta$-catenin, AXIN2, E-cadherin, ZO-1, and nuclear $\beta$-catenin and AXIN2 were analyzed by western blot. (D) Overexpression of WNT4 enhanced the TCF/LEF transcription activity. ( $E$ and F) Tumor cell metastasis assay was performed in vivo; the ratio of liver weight to body weight and the number of liver metastases in the Scramble group are much higher than the WNT4-shRNA group ( $n=7)$. Scale bar, 50 $\mu \mathrm{m}$. ( $\mathrm{G}$ and $\mathrm{H}$ ) A subcutaneous xenograft model in nude mice was used to identify if WNT4 can promote the proliferation of colon cancer cells (LoVo cell line, $n=7$; SW480 cell line, $n=5$ ). However, no significant difference was observed. (G) Scale bar, $20 \mu \mathrm{m}$. (H) Scale bar, $50 \mu \mathrm{m}$. Data are presented as the mean \pm SD. Two-tailed Student's t test was used for statistical analyses. ${ }^{*} \mathrm{P}<0.05$. 

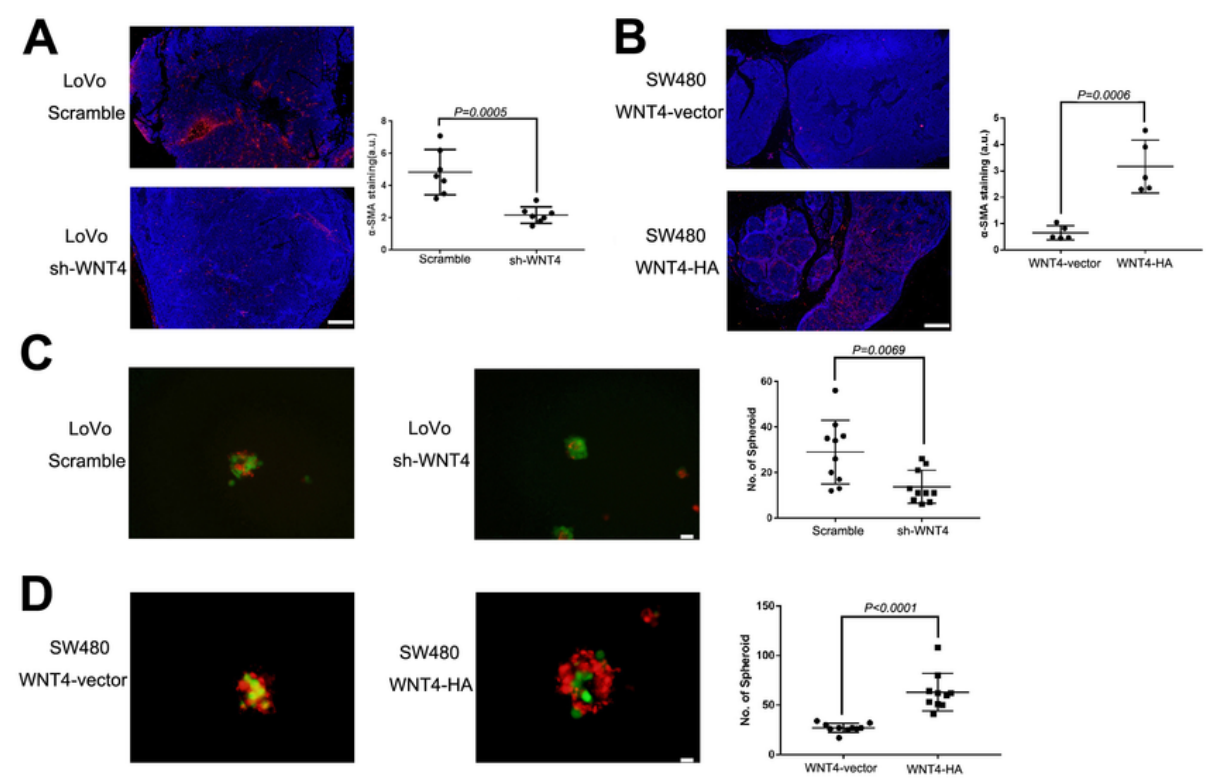

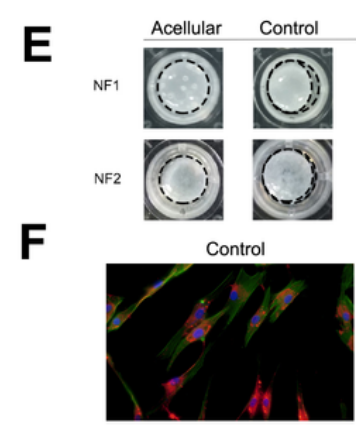

TGF $\beta(10 \mathrm{ng} / \mathrm{mL})$

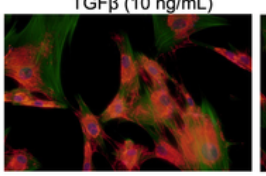

H
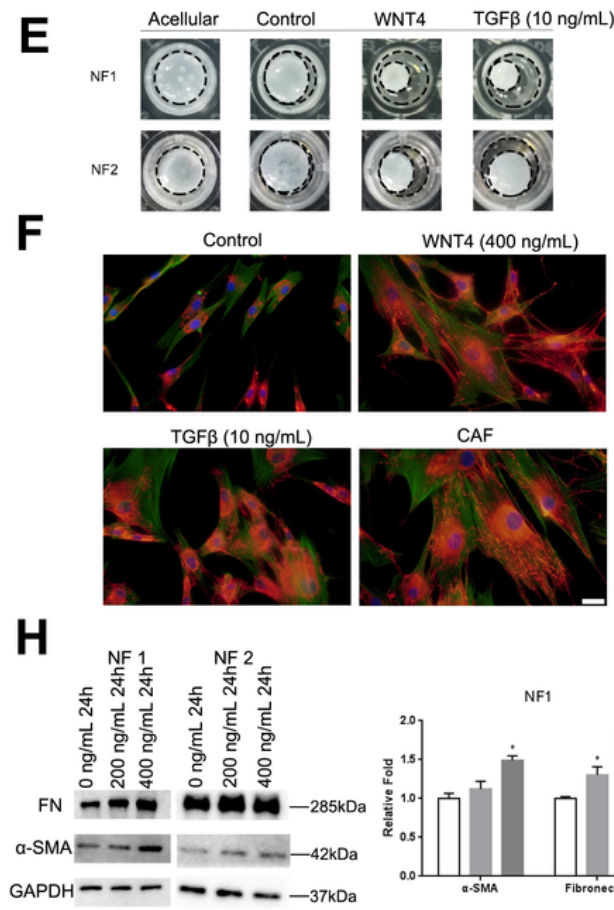

WNT4 $(400 \mathrm{ng} / \mathrm{mL})$

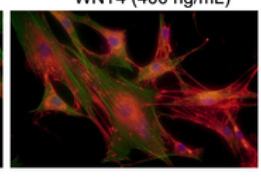

CAF
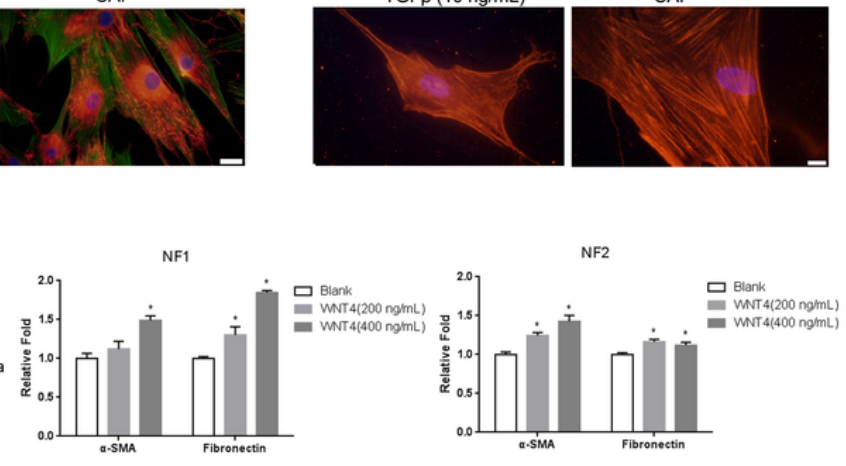

Figure 4

WNT4 promotes fibroblast recruitment and activation. (A and B) The expression of infiltrating a-SMA-positive CAFs (red) in different groups: LoVo-Scramble or LoVo-shWNT4 ( $n=7)$, SW480-WNT4-vector or SW480-WNT4-HA ( $n=5)$. Scale bar, $200 \mu \mathrm{m}$. (C and D) Typical heterospheroids formed in different groups: LoVo-Scramble or LoVo-shWNT4, SW480-WNT4- vector or SW480-WNT4- HA. CAF, red; CRC cells, green; Scale bar, $20 \mu \mathrm{m}$. (E) Stronger gel contraction was observed after the NF\#1 and NF\#2 treatment with WNT4 (400 ng/mL). ( $F$ and G). Increased expression of FN and a-SMA were observed by immunofluorescence. CAFs and NFs treated with TGF- $\beta(10 \mathrm{ng} / \mathrm{mL})$ were regarded as positive controls. (F) fibronectin-red, phalloidin-green, scale bar, $50 \mu \mathrm{m}$. (G) a-SMA-red, scale bar, $20 \mu \mathrm{m}$. (H) Increased expression of FN and a-SMA were observed by western blotting analysis. A two-tailed Student's t test was used for statistical analyses. Measurement data were presented as the mean $\pm \mathrm{SD}$. ${ }^{*} \mathrm{P}<0.05$. 
A
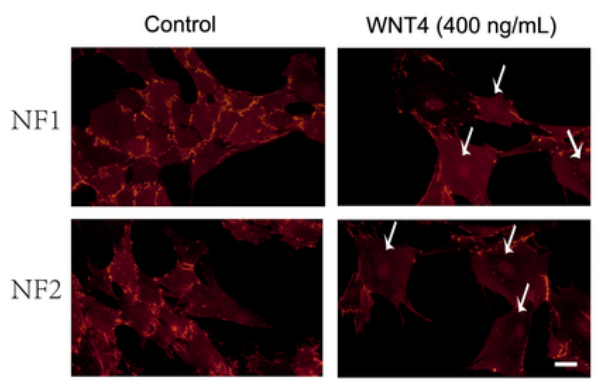

C

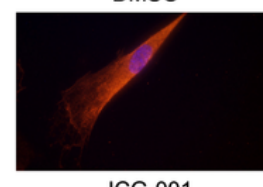

ICG-001

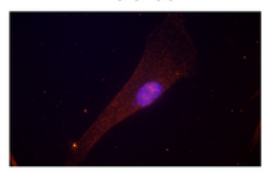

DMSO+WNT4

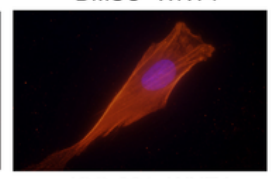

ICG-001+WNT4

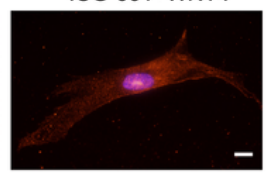

B
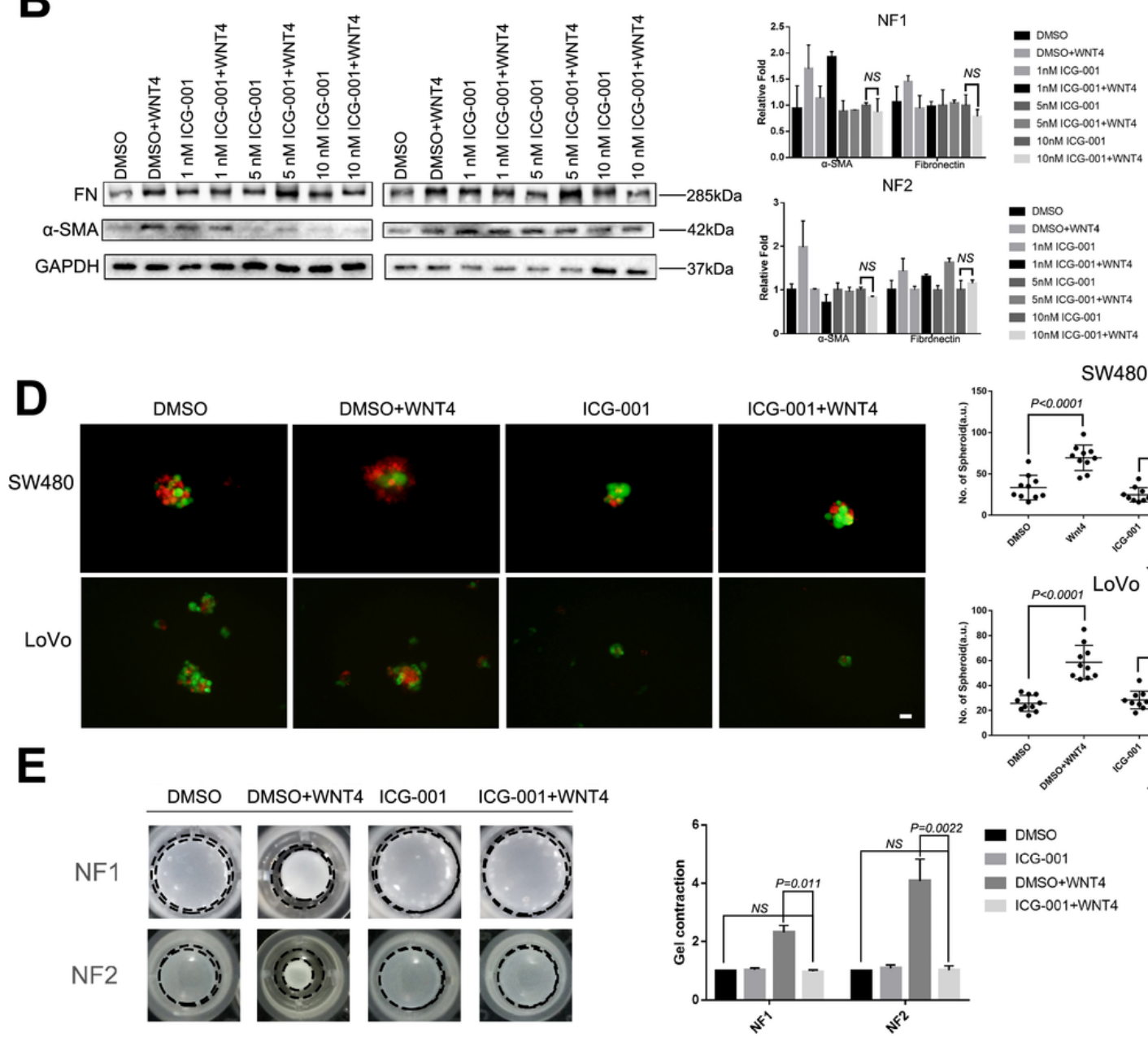

Figure 5

WNT4 signaling in fibroblasts. (A) $\beta$-catenin translocation from the cytoplasm to the nucleus in the NF\#1, NF\#2 treated with WNT4 protein (400 ng/ml, $24 \mathrm{~h}$ ). Scale bar, $50 \mu \mathrm{m}$. (B and C) ICG-001 completely blocked elevation of a-SMA and fibronectin at $10 \mu \mathrm{M}$ was detected by western blot. Scale bar, $20 \mu \mathrm{m}$. (D and E) The effect of WNT4 on the heterospheroids formation and gel contraction ability also being completely inhibited by ICG-001 $(10 \mu \mathrm{M})$. Scale bar, $20 \mu \mathrm{m}$. Measurement data were presented as the mean $\pm \mathrm{SD}$. ${ }^{*} \mathrm{P}<0.05$; NS, no significance. 

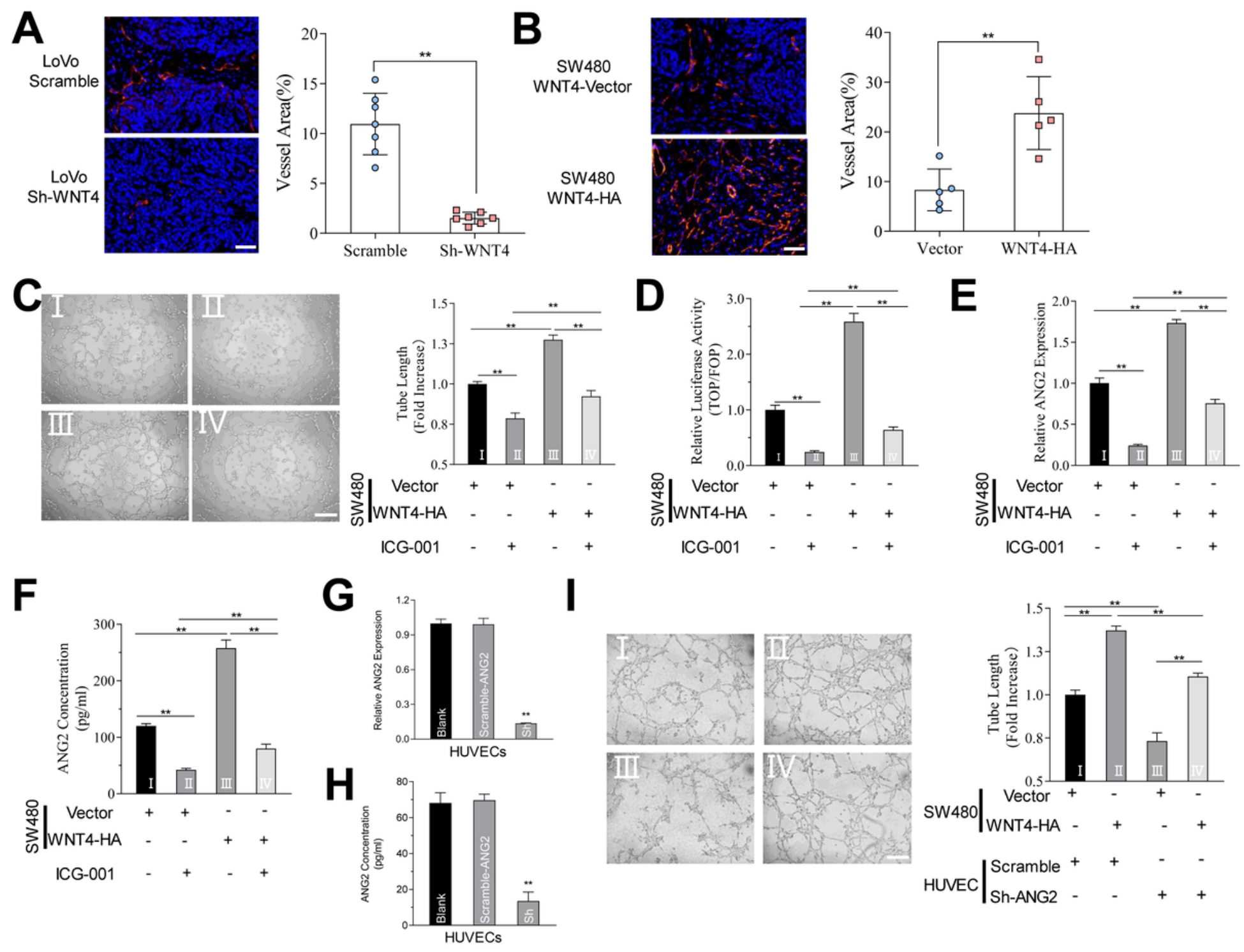

Figure 6

WNT4 promote angiogenesis in CRC. (A and B) The expression of CD31 (red) in different groups: LoVo-Scramble or LoVoshWNT4 (A), SW480-WNT4-vector or SW480-WNT4-HA. Scale bar, $50 \mu \mathrm{m}$. (C) The ability of tube formation was improved obviously when the HUVECs were treated with CM from SW480 cells transfected with WNT4-HA than that fromSW480 transfected WNT4-vector, and it could be restrained by the $\beta$-catenin/TCF inhibitor ICG-001 (10 $\mu \mathrm{M})$. Scale bar, $100 \mu \mathrm{m}$. (D) TOP/FOP-Flash reporter was used to detect whether the WNT pathway is activated. (E and F) The mRNA level of ANG2 and its concentrations were detected by RT-qPCR and ELISA in different treated HUVECs. (G and H) The mRNA level of ANG2 in cells and condition medium of HUVECs transfected with negative control shRNA (Scramble) and sh-ANG2 were detected by RTqPCR. (I) The ability of tube formation promoted by high WNT4 expression were significantly inhibited due to low ANG2 expression. Scale bar, $100 \mu \mathrm{m} .{ }^{* \star} \mathrm{P}<0.01$. 

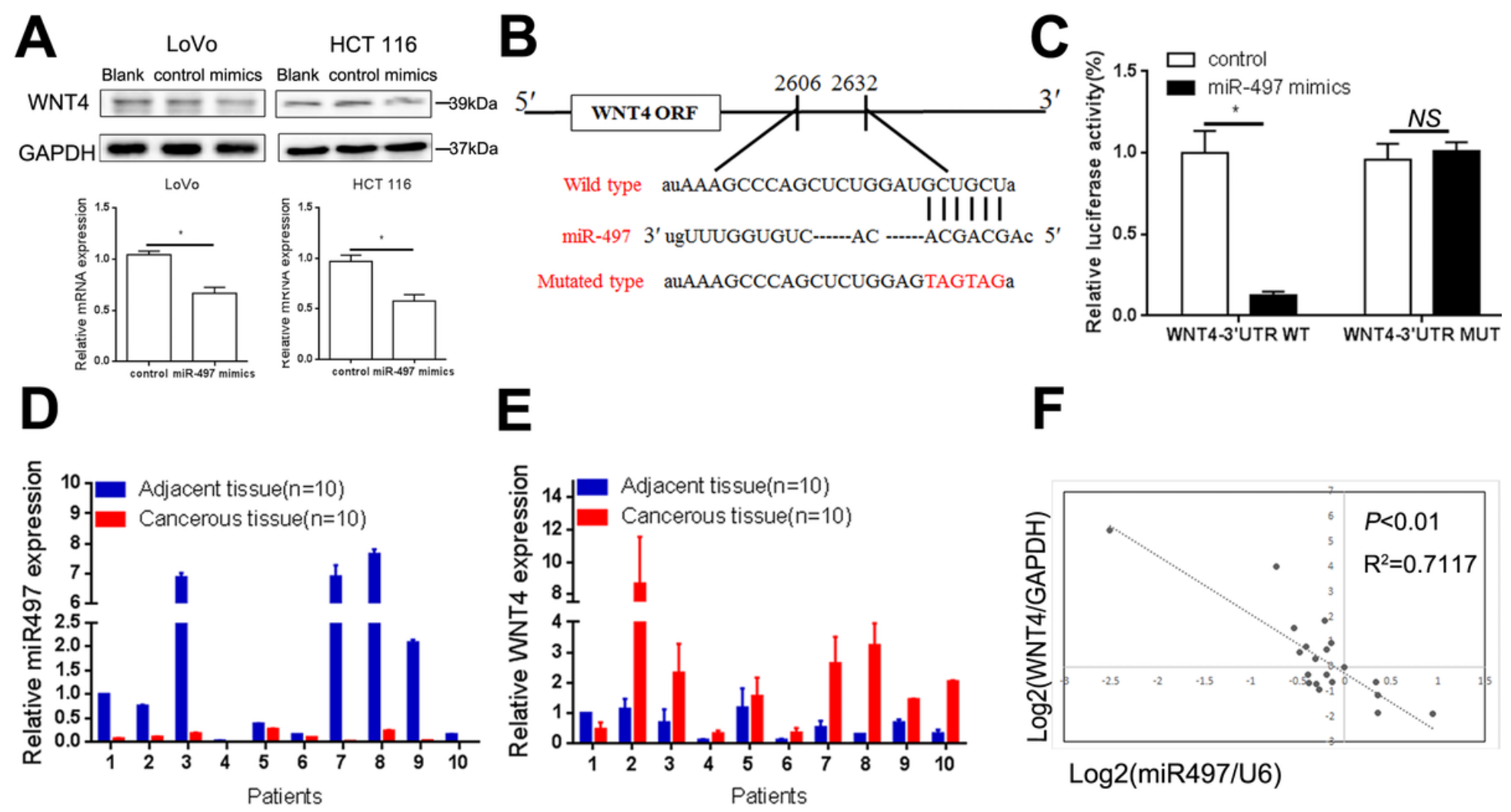

Figure 7

(A). Western blotting assays and RT-qPCR showed the protein and mRNA expression of WNT4 in CRC cells after transfecting with miR-497 mimics. (B) The target sites of miR-497 in 3'-UTR of WNT4 are shown as a schematic representation. (C) Wildtype or mutant 3'-UTR constructs of WNT4 were cloned into PMIR-REPORT ${ }^{\mathrm{TM}}$ vectors, respectively, and co-transfected with miR497 mimics in LoVo cells. (D and E) The mRNA level of miR-497 and WNT4 in the paired CRC tissues and adjacent tissues. (F) An inverse correlation was found between miR-497 expression and WNT4 in 10 paired CRC tissues and adjacent normal tissues (Spearman's correlation, $\mathrm{P}<0.01, \mathrm{R} 2=0.7117$ ).

\section{Supplementary Files}

This is a list of supplementary files associated with this preprint. Click to download.

- Additionalfile4.png

- Additionalfile3.docx

- Additonalfile2.docx

- Additionalfile1.docx 